\title{
An epidemiologic study of antimicrobial resistance of Staphylococcus species isolated from equine samples submitted to a diagnostic laboratory
}

Ronita Adams ${ }^{1}$, Jackie Smith², Stephen Locke², Erica Phillips², Erdal Erol ${ }^{2}$, Craig Carter ${ }^{2}$ and Agricola Odoi ${ }^{1 *}$ (D)

\begin{abstract}
Background: Antimicrobial resistance limits traditional treatment options and increases costs. It is therefore important to estimate the magnitude of the problem so as to provide empirical data to guide control efforts. The aim of this study was to investigate the burden and patterns of antimicrobial resistance (AMR) among equine Staphylococcus samples submitted to the University of Kentucky Veterinary Diagnostic Laboratory (UKVDL) from 1993 to 2009. Retrospective data of 1711 equine Staphylococcus samples submitted to the UKVDL during the time period 1993 to 2009 were included in the study. Antimicrobial susceptibility testing, that included 16 drugs, were performed using cultures followed by the Kirby-Bauer disk diffusion susceptibility test. The proportion of resistant isolates by animal breed, species of organism, sample source, and time period were computed. Chi-square and Cochran-Armitage trend tests were used to identify significant associations and temporal trends, respectively. Logistic regression models were used to investigate predictors of AMR and multidrug resistance (MDR).
\end{abstract}

Results: A total of $66.3 \%$ of the isolates were resistant to at least one antimicrobial, most of which were Staphylococcus aureus (77.1\%), while 25.0\% were MDR. The highest level of resistance was to penicillins (52.9\%). Among drug classes, isolates had the highest rate of AMR to at least one type of $\beta$-lactams (49.2\%), followed by aminoglycosides (30.2\%). Significant $(p<0.05)$ associations were observed between odds of AMR and horse breed, species of organism and year. Similarly, significant $(p<0.05)$ associations were identified between odds of MDR and breed and age. While some isolates had resistance to up to 12 antimicrobials, AMR profiles featuring single antimicrobials such as penicillin were more common than those with multiple antimicrobials.

Conclusion: Demographic factors were significant predictors of AMR and MDR. The fact that some isolates had resistance to up to 12 of the 16 antimicrobials assessed is quite concerning. To address the high levels of AMR and MDR observed in this study, future studies will need to focus on antimicrobial prescription practices and education of both practitioners and animal owners on judicious use of antimicrobials to slow down the development of resistance.

Keywords: Staphylococcus, Antimicrobial resistance, Multidrug resistance, Horses, Equine, Kentucky, Burden, Predictors, Logistic regression model

\footnotetext{
* Correspondence: aodoi@utk.edu

${ }^{1}$ Department of Biomedical and Diagnostic Sciences, University of Tennessee,

College of Veterinary Medicine, 2407 River Dr., Knoxville, TN 37996, USA

Full list of author information is available at the end of the article
}

(c) The Author(s). 2018 Open Access This article is distributed under the terms of the Creative Commons Attribution 4.0 International License (http://creativecommons.org/licenses/by/4.0/), which permits unrestricted use, distribution, and reproduction in any medium, provided you give appropriate credit to the original author(s) and the source, provide a link to the Creative Commons license, and indicate if changes were made. The Creative Commons Public Domain Dedication waiver (http://creativecommons.org/publicdomain/zero/1.0/) applies to the data made available in this article, unless otherwise stated. 


\section{Background}

The development of antimicrobial agents has been one of the most critical advances in both human and veterinary medicine within the last century. However, due to a combination of factors, but most notably to the rise in the use of antimicrobials for treating both human and domestic species, antimicrobial resistance has become a global scientific and public health concern in both human and veterinary medicine $[1,2]$. The quantity of antimicrobials used in both human and veterinary medicine as well as in aquaculture have contributed to the selection for antimicrobial resistance [3]. High rates of antimicrobial resistant bacterial infections increase morbidity, be it to a single agent, or multiple drug classes, hindering the ability to effectively treat infections. As a result, both morbidity and mortality of antimicrobial resistant infections have increased in affected populations [1]. Identification of the resistance profiles of microorganisms is a critical step in understanding antimicrobial resistance and is useful in providing information to guide treatment options and to combat the problem.

According to the World Health Organization, the frequency of resistance to first-line drugs that have traditionally been used to treat infections caused by Staphylococcus has increased globally [4]. Unfortunately, this resistance is not limited to human medicine, but is being seen more frequently in domestic species, and in equine medicine in particular [1]. Although the widespread use of antimicrobials among equine species in the U.S. has been addressed in multiple forums, the epidemiology of antimicrobial resistance in bacteria found in horses has not been assessed [2]. Identifying and describing the burden of antimicrobial resistance among domestic species has become even more important due to evidence of potential cross transmission of certain bacteria between humans and domestic species [5]. Both the Centers for Disease Control and Prevention (CDC) and the United States Department of Agriculture (USDA) have reported such findings in past years $[6,7]$. Outcomes from these investigations found evidence of a potential zoonotic transfer of Staphylococcus bacteria and/or their genetic material between healthy humans and horses $[6,8]$. Other reports suggest that resistant Staphylococcus infections in domestic animals may contribute to transmission seen in human contacts [9].

Understanding the burden of antimicrobial resistant Staphylococcus infections in horses is critical in not only being able to understand the risk to those in immediate contact with these animals, but also in effectively providing information to guide efforts for the development of antimicrobial stewardship programs. Although a number of studies have investigated mainly methicillin-resistant S. aureus in horses [10-14], many other Staphylococcus species not only exhibit resistance to antimicrobials, but are clinically relevant to understanding the epidemiology of antimicrobial resistance in horses and its zoonotic spread to humans [2]. Thus, the objective of this study was to estimate the proportion of antimicrobial resistant staphylococcal isolates among equine samples submitted to the University of Kentucky Veterinary Diagnostic Laboratory between 1993 and 2009 and to identify potential predictors of antimicrobial resistance and multidrug resistance.

\section{Methods}

\section{Data sources, preparation \& study area}

Laboratory records of all samples from horses submitted to the University of Kentucky Veterinary Diagnostic Laboratory were included in this study. The records included a combination of antimicrobial sensitivity test results and animal demographic information. For the isolation of bacteria, specimens were cultured on blood agar and eosin methylene blue agar plates at $37{ }^{\circ} \mathrm{C}$ in $5-10 \%$ $\mathrm{CO}_{2}$, for a minimum of $24 \mathrm{~h}$. If the specimen was from a likely contaminated site such as nasal swab, a Columbia colistin and nalidixic acid (CNA) plate with blood was also inoculated. The plates were examined for pathogenic bacteria and were incubated for an additional $24 \mathrm{~h}$ at $37^{\circ} \mathrm{C}$ in aerobic incubators and examined again for pathogenic bacteria. The criteria used for reporting a microorganism was the isolation of the microorganism in pure culture or significant numbers from specimens (as the predominate microorganism). Staphylococcus isolates were identified by using colony morphology, dark-field examination, $\beta$-hemolysis on blood agar and CNA plates, and conventional biochemical tests, including coagulase, catalase, maltose, mannitol, and trehalose. Additionally, selective and differential plates with antimicrobials and indicator were used to differentiate between $S$. aureus and S. hyicus.

Antimicrobial susceptibility testing, that included 16 drugs, were performed using Kirby-Bauer disk diffusion susceptibility test. The laboratory followed procedures of the Clinical Laboratory Standards Institute (CLSI) testing and classification to determine the susceptibility of isolates [15-19]. Sizes of the zones of inhibition were measured and interpreted as susceptible, intermediate, or resistant. Sizes of zones of susceptible and resistant in millimeters were as follows: bacitracin $(\geq 13, \leq 8)$, cephalothin $(\geq 18$, $\leq 14)$, erythromycin $(\geq 21, \leq 15)$, neomycin $(\geq 17, \leq 12)$, kanamycin $(\geq 18, \leq 13)$, streptomycin $(\geq 15$, $\leq 11)$, oxacillin $(\geq 13, \leq 10)$, lincomycin $(\geq 19, \leq 15)$, enrofloxacin $(\geq 21, \leq 17)$, amoxicillin/clavulanic acid $(\geq 20, \leq 19)$, nitrofurantoin $(\geq 17, \leq 14)$, gentamicin $(\geq 15$, $\leq 12)$, novobiocin $(\geq 17, \leq 14)$ penicillin $(\geq 28$, $\leq 19)$, tetracycline $(\geq 23, \leq 18)$, and trimethoprim and sulfamethoxazole $(\geq 16, \leq 10)$. Isolates were classified as either susceptible, intermediate or 
resistant based on the above classification procedure [15-19]. For the purpose of this study, only susceptible and resistant isolates were included for subsequent analyses. Only records from the state of Kentucky were included in the study.

\section{Data analysis}

All statistical analyses were performed in SAS 9.4 [20]. For the purpose of this study, the resistance status variable was reclassified into a binary outcome, resistant or susceptible. Thus, all isolates indicated as "intermediate" were not included in the analysis. Antimicrobial resistance (AMR) was defined as resistance to at least one antimicrobial. Additionally, multi-drug resistance (MDR) was defined as resistance to three or more antimicrobial classes [21]. The proportion of resistant isolates and 95\% confidence intervals were computed by breed, sex, age, sample source, the species of Staphylococcus, antimicrobial agent, year (which was scaled by subtracting 1993 from each year), season and month. Season was classified as follows: summer (June-August), fall (SeptemberNovember), winter (December-February), and spring (March-May). All specimen types that had frequencies of less than $1 \%$ were combined into a category called "Other". These were too many to list. Similarly, breeds with frequencies less than $1 \%$ were classified as "other breeds" and included Appaloosa, Belgian, Burro, Clydesdale, Donkey, Draft, French Warmblood, Hanover, Miniature Horse, Missouri Fox Trotter, Morgan, Other, Paint, Palomino, Percheron, and Pony.

Temporal graphs were generated in excel to visualize the temporal patterns of resistance. In addition, the Cochran-Armitage Trend test was used to identify significant temporal trends. Simple and multivariable logistic regression models were used to investigate if AMR had significant associations with breed, sex, age, sample source, species of Staphylococcus organism, year, season, and month. The model building process was done in two steps. In the first step, simple logistic regression models were fitted with "AMR, $(1=$ Resistant, 0 = Susceptible)" as the outcome and each of the variables in Table 1 as the explanatory variables. Variables with $p$-values less than 0.15 were considered for inclusion in the multivariable logistic regression model that was used in the second step. During this 2nd step, the multivariable logistic regression model was fitted using a manual backwards selection procedure. Confounding was assessed by comparing the change in parameter estimate of the variables in the model with and without the suspected confounding variable. A $20 \%$ change in the estimate of any of the variables already in the model was considered to be indicative of a confounder that was then retained in the final model. Odds ratios and their corresponding 95\% confidence intervals were computed for all variables included in the final model. Goodness-of-fit of the final model was assessed using the Hosmer-Lemeshow goodness-of-fit test. No evidence of lack of fit was found. Steps 1 and 2 for the process above were repeated to investigate predictors of multidrug resistance (MDR). In this model, the outcome variable used was "MDR, $(1=$ Multidrug Resistant $/ 0=$ Not Multidrug Resistant)". Again, Goodness-of-fit of the final model was assessed using the Hosmer-Lemeshow goodness-of-fit test. No evidence of lack of fit was found.

\section{Results}

\section{Summary statistics}

A total of 1711 samples, from 26 horse breeds, were included in the study. The most common breeds were Thoroughbreds (74.3\%) followed by Tennessee Walking Horses (5.6\%) (Table 1). Overall, more samples were submitted from female horses (83.7\%) than male horses (16.3\%) (Table 1). Similarly, horses $>4$ years old contributed the highest proportion of samples (46.0\%), followed by aborted fetuses (22.6\%) and those $<1$ year old (19.7\%) (Table 1). Additionally, samples testing positive for coagulase negative Staphylococcus were most frequent (47.8\%), followed by coagulase positive Staphylococcus aureus (40.3\%). S. hyicus was the least frequent (4.4\%).

Overall, $66.3 \%$ of the isolates were resistant to at least one antimicrobial. Of the samples with known breed information, the highest proportion of resistant isolates was from Thoroughbreds (70.5\%) followed by the Standardbreds (68.6\%) and Arabians (68.4\%), while the lowest proportion of resistance was seen in mixed breeds (40.0\%) (Table 1). Standardbreds had the highest proportion of MDR isolates (37.1\%), followed by Thoroughbreds (31.1\%), and Quarter Horse (18.3\%). The lowest proportion of MDR was in the Tennessee Walking Horse (3.4\%) (Table 1). Although females seemed to have a slightly higher level of AMR (68.1\%) than males (64.0\%), these differences were not statistically significant. However, the same does not apply to the levels of MDR between the sexes. In fact, males had a markedly higher proportion of MDR (32.9\%) than females (25.4\%) (Table 1).

Foals $(<1$ years old) showed the highest levels of AMR (75.9\%), followed by horses $2-4$ years old (67.3\%), and yearlings ( $1-2$ years old) $(65.6 \%)$. Adult horses (> 4 years old) had the lowest levels of antimicrobial resistance $(60.0 \%)$ (Table 1). Foals again showed the highest levels of MDR (37.6\%) when compared with other age groups (Table 1). MDR for horses 2-4 years old (28.9\%) and those $1-2$ years old $(18.8 \%)$ were again the next highest. The highest proportion of AMR was observed among Staphylococcus aureus isolates (77.1\%) followed by coagulase negative Staphylococcus strains (60.1\%) (Table 1). Similarly, Staphylococcus aureus (38.3\%) again 
Table 1 Distribution and antimicrobial resistance of equine Staphylococcus samples submitted to the University of Kentucky veterinary diagnostic laboratory, 1993-2009

\begin{tabular}{|c|c|c|c|c|c|c|c|c|c|}
\hline Variable & $\begin{array}{l}\text { No. of samples } \\
\text { tested }\end{array}$ & $\begin{array}{l}\text { Percentage of } \\
\text { samples tested }(\%)\end{array}$ & $95 \% \mathrm{Cl}^{\mathrm{a}}$ & $\mathrm{AMR}^{\mathrm{b}}$ samples & $\operatorname{AMR}^{\mathrm{b}, \mathrm{f}}(\%)$ & $95 \% \mathrm{Cl}^{\mathrm{a}}$ & MDR $^{\mathrm{C}}$ samples & $\mathrm{MDR}^{\mathrm{c}, \mathrm{g}}(\%)$ & $95 \% \mathrm{Cl}^{\mathrm{a}}$ \\
\hline Breed & $n=1577$ & & & $n=1046$ & & & & & \\
\hline Arabian & 19 & 1.2 & $0.7,1.7$ & 13 & 68.4 & $43.5,87.4$ & 4 & 21.1 & $6.1,45.6$ \\
\hline $\begin{array}{l}\text { American } \\
\text { Saddlebred }\end{array}$ & 63 & 4.0 & $3.0,5.0$ & 34 & 54.0 & $40.9,66.6$ & 10 & 15.9 & $7.9,27.3$ \\
\hline Mixed breed & 30 & 1.9 & $1.2,2.6$ & 12 & 40.0 & $22.7,59.4$ & 3 & 10.0 & $2.1,26.5$ \\
\hline Quarter horse & 60 & 3.8 & $2.9,4.8$ & 28 & 46.7 & $33.8,60.0$ & 11 & 18.3 & $9.5,30.4$ \\
\hline $\begin{array}{l}\text { Rocky Mountain } \\
\text { Saddlebred }\end{array}$ & 16 & 1.0 & $0.5,1.5$ & 7 & 43.8 & $19.8,70.1$ & 1 & 6.3 & $0.2,30.2$ \\
\hline Standardbred & 35 & 2.2 & $1.6,3.1$ & 24 & 68.6 & $50.7,83.2$ & 13 & 37.1 & $21.5,55.1$ \\
\hline Thoroughbred & 1172 & 74.3 & $72.2,76.5$ & 826 & 70.5 & $67.8,73.1$ & 365 & 31.1 & $28.5,33.9$ \\
\hline $\begin{array}{l}\text { Tennessee } \\
\text { Walking Horse }\end{array}$ & 88 & 5.6 & $4.5,6.7$ & 46 & 52.3 & $41.4,63.0$ & 3 & 3.4 & $8.5,75.5$ \\
\hline Other breeds & 94 & 6.0 & $4.8,7.3$ & 56 & 59.6 & $49.0,69.6$ & 9 & 9.6 & $4.5,17.4$ \\
\hline Sex & $n=1377$ & & & $n=928$ & & & & & \\
\hline Female & 1152 & 83.7 & $81.6,85.6$ & 784 & 68.1 & $65.3,70.7$ & 293 & 25.4 & $22.9,28.1$ \\
\hline Male & 225 & 16.3 & $14.4,18.4$ & 144 & 64.0 & $57.4,70.3$ & 74 & 32.9 & $26.6,39.5$ \\
\hline Age Groups & $n=717$ & & & $n=459$ & & & & & \\
\hline$>4$ years & 330 & 46.0 & $42.4,49.7$ & 198 & 60.0 & $54.5,65.3$ & 68 & 34.3 & $16.4,25.4$ \\
\hline $2-4$ years & 52 & 7.3 & $5.5,9.4$ & 35 & 67.3 & $52.9,79.7$ & 15 & 28.9 & $17.1,43.1$ \\
\hline $1-2$ years & 32 & 4.5 & $3.0,6.0$ & 21 & 65.6 & $46.8,81.4$ & 6 & 18.8 & $7.2,36.4$ \\
\hline$<1$ year & 141 & 19.7 & $16.8,22.6$ & 107 & 75.9 & $68.0,82.7$ & 53 & 37.6 & $29.6,46.1$ \\
\hline $\begin{array}{l}\text { Aborted fetus } \\
\text { (0 years) }\end{array}$ & 162 & 22.6 & $19.5,25.7$ & 98 & 60.5 & $52.5,68.1$ & 35 & 21.6 & $15.5,28.8$ \\
\hline Species of organism & $n=1711$ & & & $n=1131$ & & & & & \\
\hline $\operatorname{CoNS}^{d}$ & 817 & 47.8 & $45.4,50.1$ & 491 & 60.1 & $56.7,63.5$ & 163 & 20.0 & $17.3,22.9$ \\
\hline $\begin{array}{l}\text { Staphylococcus } \\
\text { aureus }\end{array}$ & 689 & 40.3 & $37.9,42.6$ & 531 & 77.1 & $73.7,80.2$ & 264 & 38.3 & $34.7,42.1$ \\
\hline $\begin{array}{l}\text { Staphylococcus } \\
\text { hyicus }\end{array}$ & 75 & 4.4 & $3.4,5.4$ & 31 & 41.3 & $30.1,53.3$ & 1 & 1.3 & $0.03,7.2$ \\
\hline $\begin{array}{l}\text { Staphylococcus } \\
\text { intermedius }\end{array}$ & 130 & 7.6 & $6.3,8.9$ & 78 & 60.0 & $51.1,68.5$ & 16 & 12.3 & $7.2,19.2$ \\
\hline
\end{tabular}

${ }^{\mathrm{a}} 95 \%$ Confidence Interval

${ }^{\mathrm{b}} \mathrm{AMR}$ : Antimicrobial Resistance

cMDR: Multidrug Resistance

${ }^{\mathrm{d}}$ Coagulase negative Staphylococcus

'The denominators are the number of samples tested for each variable and vary (e.g. breed = 1577; sex = 1377; etc) due to missing data

${ }^{f}$ The denominators for the percentage of AMR are the number of samples tested per row

${ }^{9}$ The denominators for the percentage of MDR are the number of samples tested per row

had the highest levels of MDR, followed by coagulase negative Staphylococcus strains (20.0\%) (Table 1).

\section{Distribution of resistance across antimicrobials}

Overall, 16 antimicrobials from 10 antimicrobial classes were examined in this study (Table 2). Highest proportions of AMR isolates were seen among $\beta$-lactams (49.2\%), with more isolates exhibiting resistance to Penicillin $(52.9 \%)$ than oxacillin (15.6\%) (Table 2 and Fig. 1). The drug class with the second highest proportion of AMR isolates was aminoglycosides (30.2\%)
(Fig. 1), with $28.9 \%$ and $22.8 \%$ of the isolates exhibiting resistance to Kanamycin and Gentamicin, respectively (Table 2). As for MDR, $\beta$-Lactams again had the highest levels $(23.5 \%)$ of isolates that were MDR followed by Aminoglycosides (22.1\%) (Table 2 and Fig. 1). Although the majority of resistant isolates (51.3\%) were only resistant to 1 or 2 antimicrobial classes, $13.4 \%$ of the resistant isolates were resistant to 5 antimicrobial classes.

Of the isolates that were found to be MDR, 8.0\% were resistant to 9 antimicrobials (Amoxycillin/clavulanic acid, Erythromicin, Gentamicin, Kanamycin, Oxacillin 
Table 2 Distribution of antimicrobial resistance categorized by antimicrobial class among equine Staphylococcus samples submitted to the University of Kentucky veterinary diagnostic laboratory, 1993-2009

\begin{tabular}{|c|c|c|c|c|c|c|c|}
\hline Antimicrobial class & Drug & AMR $^{a}$ samples & $\mathrm{AMR}^{\mathrm{a}} \%$ & $95 \% \mathrm{Cl}^{\mathrm{b}}$ & MDR ${ }^{C}$ samples & $\mathrm{MDR}^{\mathrm{C}} \%$ & $95 \% \mathrm{Cl}^{\mathrm{b}}$ \\
\hline \multirow[t]{5}{*}{ Aminoglycosides } & & $516 / 1710$ & 30.2 & $28.0,32.4$ & $377 / 1710$ & 22.1 & $20.1,24.1$ \\
\hline & Neomycin & $53 / 1582$ & 3.4 & $2.5,4.4$ & $46 / 1582$ & 2.9 & $2.1,3.9$ \\
\hline & Kanamycin & $486 / 1682$ & 28.9 & $26.7,31.1$ & $369 / 1682$ & 21.9 & $20.0,24.0$ \\
\hline & Streptomycin & $59 / 287$ & 20.6 & $16.0,25.7$ & $28 / 287$ & 9.8 & $6.6,13.8$ \\
\hline & Gentamicin & $369 / 1622$ & 22.8 & $20.7,24.9$ & $270 / 1622$ & 16.7 & $14.9,18.6$ \\
\hline \multirow[t]{4}{*}{$\beta$-lactams } & & $841 / 1710$ & 49.2 & $46.8,51.6$ & $402 / 1710$ & 23.5 & $21.5,25.6$ \\
\hline & Penicillin & $814 / 1539$ & 52.9 & $50.4,55.4$ & 396/1539 & 25.7 & $23.6,28.0$ \\
\hline & Oxacillin & $254 / 1634$ & 15.6 & $13.8,17.4$ & $235 / 1634$ & 14.4 & $12.7,16.2$ \\
\hline & Amoxicillin/Clavulanic Acid & $115 / 1644$ & 7.0 & $5.8,8.3$ & $107 / 1644$ & 6.5 & $5.4,7.8$ \\
\hline \multirow[t]{2}{*}{ Macrolides } & & $292 / 1668$ & 17.5 & $15.7,19.4$ & $249 / 1668$ & 14.9 & $13.3,16.7$ \\
\hline & Erythromycin & 292/1668 & 17.5 & $15.7,19.4$ & 249/1668 & 14.9 & $13.3,16.7$ \\
\hline \multirow[t]{3}{*}{ Sulfonamides } & & $463 / 1645$ & 28.2 & $26.0,30.4$ & $372 / 1645$ & 22.6 & $20.6,24.7$ \\
\hline & Sulfonamide & $488 / 1702$ & 28.7 & $26.5,30.9$ & $372 / 1702$ & 21.9 & $19.9,24.0$ \\
\hline & Trimethoprim-sulfadiazine & $330 / 1355$ & 24.4 & $22.1,26.7$ & 297/1355 & 21.9 & $19.7,24.2$ \\
\hline \multirow[t]{2}{*}{ Lincosamides } & & 28/970 & 2.9 & $1.9,4.2$ & 25/970 & 2.6 & $1.7,3.8$ \\
\hline & Lincomycin & 28/970 & 2.9 & $1.9,4.2$ & 25/970 & 2.6 & $1.7,3.8$ \\
\hline \multirow[t]{2}{*}{ Aminocoumarins } & & $141 / 1578$ & 8.9 & $7.6,10.6$ & $31 / 1578$ & 2.0 & $1.3,2.8$ \\
\hline & Novobiocin & $141 / 1578$ & 8.9 & $7.6,10.6$ & $31 / 1578$ & 2.0 & $1.3,2.8$ \\
\hline \multirow[t]{2}{*}{ Cephalosporins } & & $63 / 1711$ & 3.7 & $2.8,4.7$ & $63 / 1711$ & 3.7 & $2.8,17.1$ \\
\hline & Cephalothin & $48 / 1692$ & 2.8 & $2.1,3.7$ & $48 / 1692$ & 2.8 & $2.1,3.7$ \\
\hline \multirow[t]{2}{*}{ Fluoroquinolones } & & $1 / 25$ & 4.0 & $0.1,20.4$ & $1 / 25$ & 4.0 & $0.1,20.4$ \\
\hline & Enrofloxacin & $1 / 24$ & 4.2 & $0.1,21.1$ & $1 / 24$ & 4.2 & $0.1,21.1$ \\
\hline \multirow[t]{2}{*}{ Tetracyclines } & & $451 / 1682$ & 26.8 & $24.7,29.0$ & $326 / 1682$ & 19.4 & $17.5,21.4$ \\
\hline & Tetracycline & $451 / 1682$ & 26.8 & $24.7,29.0$ & $326 / 1682$ & 19.4 & $17.5,21.4$ \\
\hline \multirow[t]{2}{*}{ Polypeptides } & & $45 / 1649$ & 2.7 & $2.0,3.6$ & $36 / 1649$ & 2.2 & $1.5,3.0$ \\
\hline & Bacitracin & $45 / 1649$ & 2.7 & $2.0,3.6$ & $36 / 1649$ & 2.2 & $1.5,3.0$ \\
\hline
\end{tabular}

${ }^{\mathrm{a} A M R}$ : Antimicrobial Resistance

${ }^{\mathrm{b}} 95 \%$ Confidence Interval

${ }^{c}$ MDR: Multidrug Resistance

(Methicillin), Penicillin, Sulfonamides, Tetracycline, and Trimethoprim-sulfadiazine) belonging to 5 antimicrobial classes (Aminoglycosides, $\beta$-Lactams, Macrolides, Sulfonamides and Tetracyclines) (Table 3). Another 7.0\% were resistant to the same profile of antimicrobials except Erythromycin. In fact, $46.0 \%$ of the isolates that were MDR, and had a sample size greater than 10, had resistance profiles that contained penicillin, kanamycin, sulfonamides, and trimethoprim-sulfadiazine (Table 3). Additionally, 34.0\% of the MDR samples with sample sizes greater than 10 showed resistance to oxacillin (Table 3).

\section{Temporal trends}

There was a significant $(p=0.023)$ decreasing temporal trend in AMR over the study period (Fig. 2). The proportions of AMR isolates were highest in 2000 (76.0\%) and reached their lowest levels by 2007 (52.4\%) (Fig. 2). On the contrary, there was an increasing temporal trend in MDR $(p=0.007)$ over the study period (Fig. 2). The proportion of MDR isolates began at its lowest point in 1993 (14.4\%) before reaching the highest level in 2000 (42.5\%) (Fig. 2).

\section{Predictors of antimicrobial resistance (AMR) and multidrug resistance (MDR)}

Species of organism, breed, age, sex, season, and year all had significant simple associations with the odds of AMR at an $\alpha=0.15$. (Table 4). Similarly, species of organism, breed, age, sex, and year had significant simple associations with the odds of MDR (Table 4). All variables found to be significant $(p \leq 0.15)$ in the AMR or 


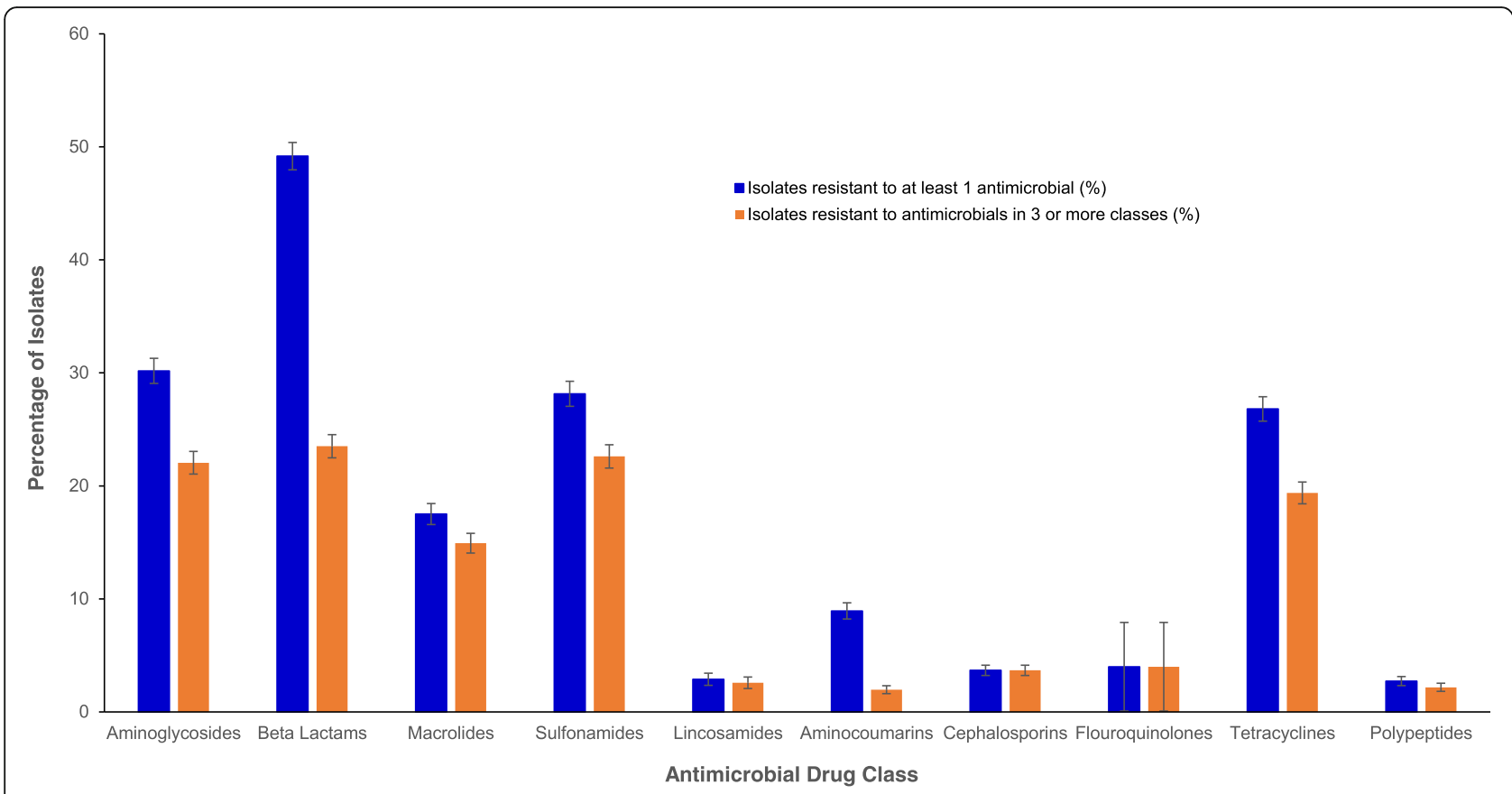

Fig. 1 Antimicrobial resistance and multidrug resistance by drug class from equine Staphylococcus samples submitted to the University of Kentucky veterinary diagnostic laboratory, 1993-2009

MDR simple models were considered for inclusion in their respective multivariable models.

Breed $(p=<0.001)$, species of organism $(p=<0.001)$ and year $(p=0.023)$ were significantly associated with the odds of antimicrobial resistant Staphylococcus infections in horses (Table 5). There was a significant $(p=<0.001)$ association between breed and AMR with Thoroughbreds having higher odds (Odds Ratio [OR] = 1.61; $95 \%$ Confidence Interval $[\mathrm{CI}]=1.07,2.42$ ) of AMR than other breeds (Table 5). Interestingly, species of organism was a significant predictor for AMR but not MDR. The odds of AMR among Staphylococcus aureus isolates was significantly $(p<0.0001)$ higher $(\mathrm{OR}=2.30$; $95 \% \mathrm{CI}=1.81,2.93)$ than that of coagulase negative Staphylococcus isolates (Table 5), while the odds of AMR among Staphylococcus hyicus isolates was significantly $(p<0.0001)$ lower $(\mathrm{OR}=0.46 ; 95 \% \mathrm{CI}=0.27$, 0.77) than that of coagulase negative Staphylococcus isolates. Year had a negative association with AMR $(\mathrm{OR}=0.97,95 \% \mathrm{CI}=0.95,1.00)$.

Table 3 Antimicrobial resistance profiles of equine resistant Staphylococcus samples submitted to the University of Kentucky veterinary diagnostic laboratory, 1993-2009

\begin{tabular}{llll}
\hline Profile & No. of samples & Percent & $95 \% \mathrm{Cl}^{\mathrm{a}}$ \\
\hline Amo-Cep-Ery-Gen-Kan-Oxa-Pen-Sul-Tet-Tri & 14 & 4.3 & $2.3,6.9$ \\
Amo-Ery-Gen-Kan-Oxa-Pen-Sul-Tet-Tri & 28 & 8.3 & $5.6,11.8$ \\
Amo-Ery-Kan-Oxa-Pen-Sul-Tet-Tri & 10 & 3.0 & $1.4,5.4$ \\
Amo-Gen-Kan-Oxa-Pen-Sul-Tet-Tri & 10 & 3.0 & $1.4,5.4$ \\
Ery-Gen-Kan-Oxa-Pen-Sul-Tet-Tri & 25 & 7.4 & $4.9,10.8$ \\
Ery-Gen-Kan-Pen-Sul-Tet-Tri & 14 & 4.2 & $2.3,6.9$ \\
Ery-Kan-Oxa-Pen-Sul-Tet-Tri & 12 & 3.6 & $1.9,6.1$ \\
Gen-Kan-Oxa-Pen-Sul-Tet-Tri & 17 & 5.0 & $3.0,8.0$ \\
Gen-Kan-Pen-Sul-Tet-Tri & 16 & 4.8 & $2.7,7.6$ \\
Gen-Kan-Pen-Sul-Tri & 10 & 3.0 & $1.4,5.4$ \\
\hline
\end{tabular}

The denominator used for each percentage was (337) after missing values were removed

Amo Amoxiillin/clavulanic acid, Cep Cephalothin, Ery Erythromycin, Gen Gentamicin, Kan Kanamycin, Oxa Oxacillin, Pen Penicillin, Sul Sulfonamide, Tet Tetracycline, Tri Trimethoprim-sulfadiazine

${ }^{\mathrm{a}} 95 \%$ Confidence Interval 


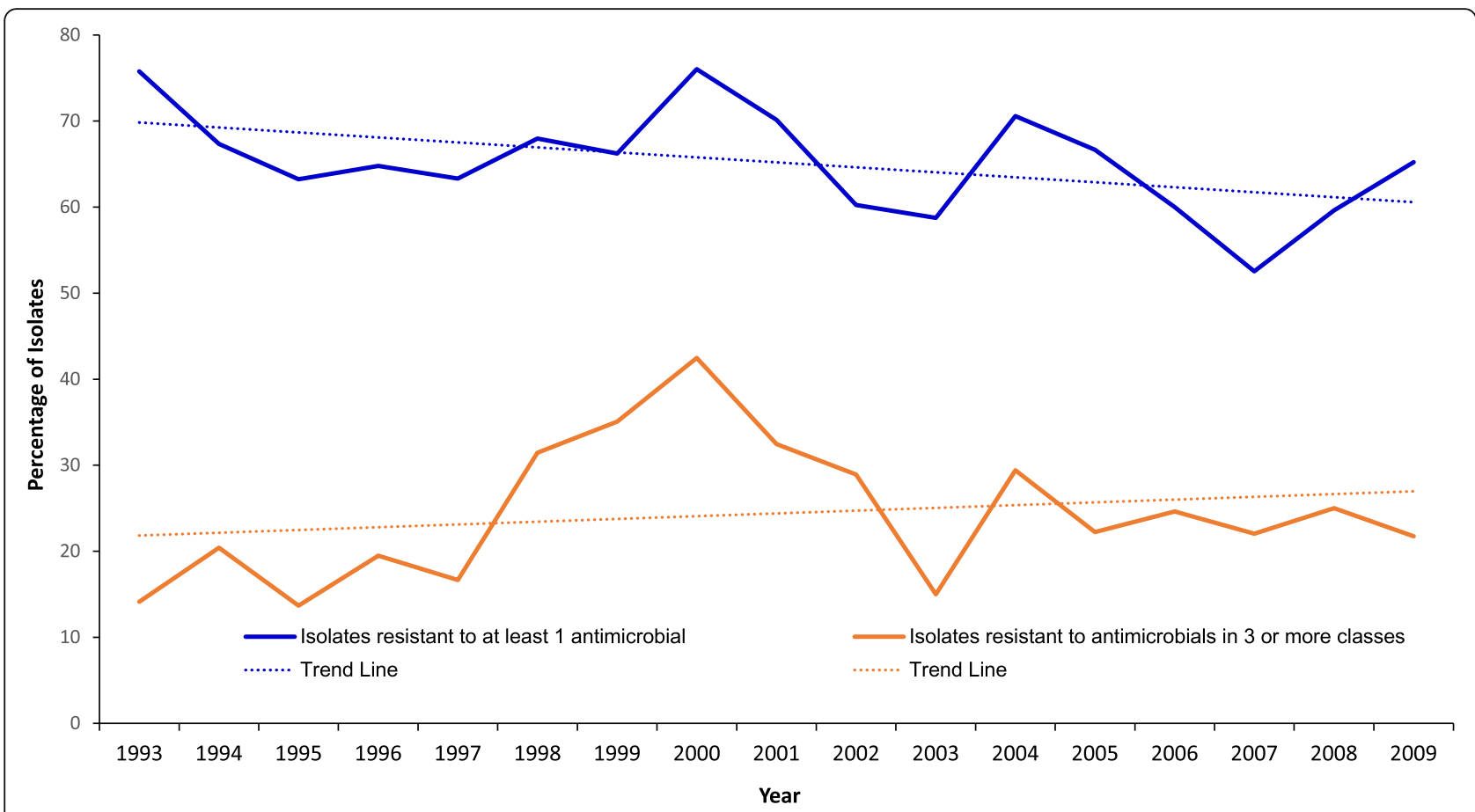

Fig. 2 Annual temporal distribution of antimicrobial resistance \& multidrug resistance from equine Staphylococcus samples submitted to the University of Kentucky veterinary diagnostic laboratory, 1993-2009

Breed $(p=<0.001)$ and age $(p=0.020)$ were significantly associated with the odds of MDR of Staphylococcus (Table 6). The odds of isolates from Standardbreds being $\mathrm{MDR}$ were over 15 times $(\mathrm{OR}=15.0 ; 95 \% \mathrm{CI}=3.7$, 60.4) higher than those of isolates from other breeds, while the odds of MDR in isolates from Thoroughbreds were almost 7 times $(\mathrm{OR}=7.0 ; 95 \% \mathrm{CI}=2.4,19.8)$ higher than that of isolates from other breeds (Table 6). The odds of MDR among isolates taken from foals $(<1$ year) were $63 \%(\mathrm{OR}=1.6 ; 95 \% \mathrm{CI}=1.0,2.6)$ higher than that of horses $>4$ years old (Table 6 ).

Table 4 Results of simple logistic models assessing predictors of antimicrobial resistance and multidrug resistance in equine Staphylococcus samples submitted to the Kentucky state diagnostic laboratory, 1993-2009

\begin{tabular}{lll}
\hline Variable & AMR $^{\mathrm{a}} P$-Value & $\mathrm{MDR}^{\mathrm{b}} P$-Value \\
\hline Breed & $<0.001$ & $<0.001$ \\
Age & 0.019 & 0.002 \\
Organism & $<0.001$ & $<0.001$ \\
Sex & 0.107 & 0.021 \\
Season & 0.083 & 0.781 \\
Month & 0.379 & 0.519 \\
Year & 0.046 & 0.001 \\
City & 0.390 & 0.146
\end{tabular}

${ }^{\text {a Antimicrobial Resistance }}$

${ }^{\mathrm{b}}$ Multidrug Resistance

\section{Discussion}

This study was designed to investigate the burden and patterns of both AMR and MDR among equine Staphylococcus samples submitted to the University of Kentucky Veterinary Diagnostic Laboratory and to

Table 5 Significant predictors of antimicrobial resistant Staphylococcus in equines from samples submitted to the Kentucky state diagnostic laboratory, 1993-2009

\begin{tabular}{llll}
\hline Variable & Odds ratio & $95 \% \mathrm{Cl}^{\mathrm{a}}$ & $P$-Value \\
\hline Breed & 1.5 & $0.5,4.3$ & $<.331$ \\
Arabian & 0.9 & $0.5,1.7$ & 0.763 \\
American Saddlebred & 0.5 & $0.2,1.2$ & 0.057 \\
Mixed Equine & 0.6 & $0.3,1.2$ & 0.091 \\
Quarter Horse & 1.5 & $0.6,3.3$ & 0.245 \\
Standard Bred & 1.6 & $1.1,2.4$ & $<.001$ \\
Thoroughbred & 0.8 & $0.5,1.5$ & 0.397 \\
Tennessee Walking Horse &. & $\cdot$ &. \\
Other Breeds & & & $<0.001$ \\
Species of Organism & 2.3 & $1.8,2.9$ & $<.001$ \\
Staphylococcus aureus & 0.5 & $0.3,0.8$ & $<.001$ \\
Staphylococcus hyicus & 1.1 & $0.7,1.8$ & 0.692 \\
Staphylococcus intermedius &. &. &. \\
Coagulase negative Staphylococcus &. & $0.95,1.00$ & 0.023 \\
\hline Year & 0.97 & &
\end{tabular}

${ }^{\mathrm{a}} 95 \%$ Confidence Interval 
Table 6 Significant predictors of multidrug resistant Staphylococcus in equines from samples submitted to the Kentucky state diagnostic laboratory, 1993-2009

\begin{tabular}{llll}
\hline Variable & Odds ratio & $95 \% \mathrm{Cl}^{\mathrm{a}}$ & $P$-value \\
\hline Breed & & & $<0.001$ \\
Arabian & 3.9 & $0.6,25.1$ & 0.159 \\
American Saddlebred & 2.5 & $0.5,12.5$ & 0.257 \\
Mixed Equine & 2.3 & $0.5,11.5$ & 0.308 \\
Quarter Horse & 2.2 & $0.5,8.7$ & 0.277 \\
Standardbred & 15.0 & $3.7,60.4$ & 0.001 \\
Thoroughbred & 7.0 & $2.4,19.8$ & 0.000 \\
Tennessee Walking Horse & 0.8 & $0.2,3.6$ & 0.730 \\
Other & $\cdot$ & $\cdot$ & $\cdot$ \\
Age & & & 0.020 \\
Aborted Fetus 0 years & 0.7 & $0.4,1.2$ & 0.171 \\
$<1$ year & 1.6 & $1.0,2.6$ & 0.042 \\
1-2 years & 1.8 & $0.6,4.9$ & 0.266 \\
2-4 years & 1.5 & $0.7,3.1$ & 0.275 \\
$>$ 4 years &. &. & $\cdot$ \\
\hline
\end{tabular}

${ }^{\mathrm{a}} 95 \%$ Confidence Interval

investigate the predictors of AMR and MDR. The findings should provide information to guide future studies and ongoing surveillance of antimicrobial resistance. The proportion of antimicrobial resistant isolates seen in this study for both coagulase negative Staphylococcus infections $(60.1 \%)$ and coagulase positive strains including S. aureus (77.1\%), S. intermedius (60.0\%), and S. hyicus (41.3\%) suggest that the levels of AMR are high for both pathogenic and non-pathogenic Staphylococcus species.

\section{Temporal trends}

The temporal patterns observed in this study are interesting as a significant decreasing temporal trend was found for AMR, while an increasing temporal trend was observed for MDR. The reasons for this are unclear. However, a University of California (U.C.), Davis study that examined temporal trends in antimicrobial susceptibility patterns in equine case records from the William R. Pritchard Veterinary Medical Teaching Hospital (VMTH) from 1979 to 2010, found statistically significant increases over time in the percentage of Staphylococcus isolates susceptible to certain antimicrobials (chloramphenicol, ceftiofur, and penicillin) [22]. It is worth noting that, the U.C. Davis study investigated multiple organisms (Pseudomonas species, Enterococcus species, E. coli, Salmonella species., Streptococcus species, Staphylococcus species and Actinobacillus species) while our study was limited to Staphylococcus species. Findings from this study suggest that despite the significant decreasing AMR temporal trends, significant increasing MDR temporal trends in this population could have a negative impact on morbidity and mortality rates attributable to MDR infections [23, 24].

\section{Antimicrobials}

There is a paucity of published literature on antimicrobial resistance in equine Staphylococcus infections. Most of the work that has been published has focused only on S. aureus and especially MRSA. Thus the lack of literature addressing resistant Staphylococcus species in horses makes comparisons between the findings of this study and others difficult. Suffice it to say that although the overall proportions of AMR isolates in this study were high, MRSA levels were much lower $(15.6 \%)$ than the percentage of MRSA (48\%) found in a similar study done in Turkey [25]. A Belgian study, by Van den Eede et al., that assessed occurrence of MRSA in equine nasal samples found similar MRSA levels (10.9\%) to those found in our study [26]. However, studies done in Australia, Canada, and Ireland that investigated Staphylococcus aureus colonization in healthy horses as well as isolation rates in horses with clinical presentation of MRSA found the percentage of AMR isolates to be much lower and ranging from $4 \%$ to $8 \%$ [27-29]. These differences could be attributed to the fact that we examined a higher number of antimicrobials and species of Staphylococcus in this study in comparison with the above studies that only investigated methicillin resistance in S. aureus.

The highest levels of resistance in this study was towards $\beta$-Lactams and Aminoglycosides. This may be due to the tendency of staphylococci to adapt to the selection pressure of antimicrobial use and become resistant to antimicrobials in general and the multiple mechanisms of resistance to aminoglycosides and $\beta$-Lactams in particular [30, 31]. These findings are comparable to those of a Swiss study which reported high levels of AMR not only to $\beta$-lactams and aminoglycosides, but to tetracyclines, lincosamides and macrolides as well when compared to other drug classes [32]. We also found the highest levels of AMR to be against penicillin (52.9\%). Much higher levels of resistance were reported from equine hospital data in Zurich, where researchers identified AMR to penicillin in both coagulase negative staphylococci and Staphylococcus aureus to be around $82 \%$ and AMR to tetracycline to be $64 \%$ [14]. High levels of resistance to both penicillin $(62.7 \%)$ and tetracycline (23.7\%) were found in a retrospective study in France that investigated Staphylococci implicated in death or euthanasia in horses [33]. The higher levels of AMR Staphylococcus infections reported in hospitals could explain the higher AMR levels from the Zurich study.

A German study looking at resistance profiles of MRSA in horses from veterinary hospitals and large animal clinics found that gentamicin resistance was high 
(85\%) and mainly associated with isolates coming from equine clinics, while the majority of the isolates from all horses in the study were resistant to tetracycline (97.5\%) and fluoroquinolones (79\%) with only $15.6 \%$ being resistant to erythromycin [34]. Our study found much lower levels of AMR to gentamicin (22.8\%), tetracycline (26.8\%) and fluoroquinolones (4.0\%), than the German study. Despite our MDR profiles containing gentamicin (16.7\%) and tetracycline (19.4\%) resistance, these levels were still not consistent with the findings of the German study. The differences in the levels of AMR and MDR seen in our study can be explained by the fact that the isolates from our study included multiple Staphylococcus species.

Of the resistant isolates in this study, 25\% were MDR. This is double the percentage of MDR (13\%) found in a Lithuanian study by Klimienè et al. [35] and a Zurich study [14] that both reported 13\% MDR. However, it is more than double that reported by Toombs-Ruane et al. (10.1\%) in New Zealand [36]. The Swiss study mentioned previously, also found that isolates were most likely to be MDR involving $\beta$-lactams, aminoglycosides, and tetracyclines [32]. That finding is similar to that of our study where the highest proportion of MDR infections involved aminoglycosides, $\beta$-lactams, sulfonamides, cephalosporins and tetracyclines. Interestingly, a recent companion animal study done in India found that not only were the incidences of Staphylococcus aureus wound infections higher in equines $(57.14 \%)$, but that there was $100 \%$ MDR against kanamycin, colistin, clindamycin, penicillin-G, cotrimoxazole and cefotaxime [37]. However, it is worth noting that the current study only focused on Staphylococcus infections in horses and not multiple companion animals as was the case in the Indian study.

\section{Antimicrobial resistance profile}

Almost half of the MDR isolates in this study had antimicrobial resistance profiles that included penicillin, kanamycin, sulfonamides, and trimethoprim-sulfadiazine. These findings are consistent with those of a similar study that found that, in isolates identified to be MDR, Staphylococcus isolates that were oxacillin resistant, were also resistant to kanamycin, gentamicin and penicillin [38]. In our study less than $1 \%$ of the isolates were resistant to 12 antimicrobials and antimicrobial resistance profiles showed MDR to occur most frequently among isolates resistant to aminoglycosides, $\beta$-lactams, tetracyclines, sulfonamides, and cephalosporins. These findings were different from those of a study done in Switzerland by Schnelleman et al., [32], where $24 \%$ of the Staphylococcus isolates were resistant to all 12 of the antimicrobials tested, while the remainder of the isolates were resistant to a number of drug classes including $\beta$-lactams, combination $\beta$-lactam- $\beta$-lactamase-inhibitors, aminoglycosides, tetracyclines, chloramphenicol, macrolides, lincosamides and/or streptogramins [32]. It is important to note that isolates from the Swiss study were obtained only from horses undergoing colic surgery. A Lithuanian study by Klimiene et al. [35], found that the Staphylococcus isolates that were MDR showed high levels of resistance to penicillin G, erythromycin or tetracycline. Similar to the findings of our study, they reported that $66.7 \%$ of the isolates showed resistances to penicillin, erythromycin, tetracycline, ciprofloxacin, and gentamicin.

\section{Distribution of resistance by host factors, species of organism and time}

Thoroughbreds had the highest proportion of antimicrobial resistance $(70.5 \%)$ in this study. This number is strikingly higher than the 5\% AMR levels found in a similar study in Japan that examined MRSA colonization and infection in thoroughbreds [12]. However, because the Japanese study only looked at MRSA in thoroughbreds, while our study was able to examine both AMR and MDR in thoroughbreds, it is difficult to make direct comparisons between the AMR levels of the two. Nonetheless, a Canadian study looked at a mixture of draft, race, pleasure, breeding, school, and show horses and found no evidence of MRSA in thoroughbreds [29]. In this study, we found that the odds of AMR in thoroughbreds was higher than that of other breeds. The higher odds of AMR in thoroughbreds could be due to the extensive movement of this particular horse breed, increasing the risk for exposure to resistant Staphylococcus strains and contributing to higher resistance levels. Another Canadian study hypothesized that frequent contact with other horses, recurring and frequent travel to different sites, and the frequent use of antimicrobials in this set of horses could be associated with increased prevalence of MRSA in show and race horses [39]. Race horses, especially thoroughbreds, are moved frequently between Canada and the United States due to the large racing industry in both countries, which makes the risk of MRSA colonization and infection more widespread than seen in other breeds [40]. Horses, and thoroughbreds, in particular, are often moved between the United States, Australia, Canada, Japan, the UK, and Ireland, increasing the risk of importing infected carrier horses [12]. This could explain the high levels of resistance seen in thoroughbreds in this study.

A significant simple association was found between age and the odds of both AMR and MDR in this study. However, a significant association was only found between MDR and age in the multivariable model with age group less than 1 year showing significantly higher odds of MDR. Many past studies have focused on foals as an important population for studies of antimicrobial susceptibility $[22,41-44]$. This is likely due to the higher susceptibility of younger animals to infection resulting in 
higher likelihood of antimicrobial treatment and hence selection for resistance. In this study Staphylococcus aureus was found to have significantly higher odds of AMR when compared with other Staphylococcus species, which is likely due to adaptability seen in S. aureus, [45], as well as the high prevalence of methicillin resistance in Staphylococcus aureus isolates, which indicates intrinsic resistance to all other $\beta$-Lactams, aminoglycosides and macrolides [46, 47].

Year was a significant predictor of AMR but not MDR in this study, where the odds of AMR isolates decreased over time. Decreases in AMR are likely due to changes in surveillance and reporting practices for resistant Staphylococcus infections, as well as adherence to sound antimicrobial prescription practices and policies. A study by Weese \& Rousseau [48] found that after implementation of both active surveillance cultures and infection control procedures to address endemic MRSA, there was a rapid decrease in the proportion of horses colonized with MRSA. The study done by Weese \& Rousseau focused on MRSA infections so direct comparisons cannot be made. However, it does indicate that appropriate control measures can affect the proportion of resistance infections observed and reported.

\section{Study limitations}

This retrospective laboratory-based study is not without limitations. Since data were not obtained using a statistical sampling technique, the study population should not be considered to be representative of the equine population in Kentucky. Only data available in the laboratory records could be investigated limiting the scope of investigation. For instance, information on past antimicrobial use was not available and therefore we could not assess its associations with levels of AMR or MDR. Furthermore, past medical history of the animals whose samples were used in this study was not reported.

\section{Conclusion}

The above limitations notwithstanding, the findings of this study provide useful information on the epidemiology of AMR and MDR in Staphylococcus infections in horses whose samples were submitted to the UKVDL. This information will be useful for guiding future primary base studies as well as efforts to address the problem. It is clear that equine Staphylococcus infections are exhibiting both AMR and MDR in horses. Factors such as breed and year are significant predictors of the odds of both AMR and MDR in this study, while species of staphylococci is also an important predictor of AMR and age of the horse was significantly associated with MDR. High levels of AMR and MDR could be indicative of problems in clinical prescription practices and procedures leading to selection for antimicrobial resistance.
This highlights the need for a more comprehensive approach to investigating the epidemiology of AMR and MDR in horses. Future studies will need to focus on improving our understanding of antimicrobial use in horses as this will allow for more informed antimicrobial stewardship programs. Moreover, AMR surveillance in horses needs to include better record keeping and lab submission information (such as pre-treatment history). More information on risk factors may be gained through primary base observational studies that can more robustly identify risk factors that might otherwise not be investigated by retrospective lab-based studies.

\section{Abbreviations \\ AMR: Antimicrobial resistance; CDC: Centers for Disease Control and Prevention; Cl: Confidence interval; CLSI: Clinical Laboratory Standards Institute; CNA: Colistin and nalidixic acid; MDR: Multidrug resistance; MRSA: Methicillin-resistant Staphylococcus aureus; SAS: Statistical analysis system; UC: University of California; UKVDL: University of Kentucky Veterinary Diagnostic Laboratory; USDA: United States Department of Agriculture; VMTH: Veterinary Medical Teaching Hospital}

\section{Acknowledgements}

The authors would like to thank the University of Kentucky Veterinary Diagnostic Laboratory for providing the study data.

\section{Funding}

The University of Tennessee, Center of Excellence in Livestock Diseases and Human Health (COE) Research Award.

\section{Availability of data and materials}

The data that support the findings of this study are available from the University of Kentucky Veterinary Diagnostic Laboratory that has legal ownership of the data. The data are not publicly available and should be requested and obtained from the above legal owner that can be reached at 1490 Bull Lea Road, Lexington, KY 40512, USA.

\section{Authors' contributions}

RA was involved in data management and performed all statistical analyses and interpretation as well as preparation of the manuscript draft. AO was involved in conceptualization of research idea, study design, data analysis and interpretation as well as extensive editing of the manuscript. JS, SL, EP, CC, and EE were involved in all laboratory analyses, provision of laboratory resources, and data curation. All authors read and approved the final manuscript.

\section{Ethics approval}

This study was approved by the University of Tennessee Institutional Animal Care \& Use Committee (IACUC). The study used retrospective laboratory records and did not involve animals. All data were handled in compliance with relevant guidelines. No field studies or experiments were conducted as part of this study and hence no informed consent was required.

\section{Consent for publication}

Not Applicable.

\section{Competing interests}

The authors declare that they have no competing interests.

\section{Publisher's Note}

Springer Nature remains neutral with regard to jurisdictional claims in published maps and institutional affiliations.

\section{Author details}

'Department of Biomedical and Diagnostic Sciences, University of Tennessee, College of Veterinary Medicine, 2407 River Dr., Knoxville, TN 37996, USA. ${ }^{2}$ University of Kentucky, Veterinary Diagnostic Laboratory, 1490 Bull Lea Rd., Lexington, KY 40511, USA. 
Received: 20 November 2017 Accepted: 29 January 2018 Published online: 05 February 2018

\section{References}

1. Bryan J, Leonard N, Fanning S, Katz L, Duggan V. Antimicrobial resistance in commensal faecal Escherichia coli of hospitalised horses. Ir Vet J. 2010;63(6):373-9.

2. Maddox TW, Clegg PD, Williams NJ, Pinchbeck GL. Antimicrobial resistance in bacteria from horses: epidemiology of antimicrobial resistance. Equine Vet J. 2015;47(6):756-65.

3. Nikaido H. Multidrug Resistance in Bacteria. Annu Rev Biochem. 2009;78: $119-46$.

4. WHO: Antimicrobial Resistrance Fact Sheet. Geneva: Who Health Organizarion.

5. Epstein JH, Price JT. The significant but understudied impact of pathogen transmission from humans to animals. Mt Sinai J Med. 2009;76(5):448-55.

6. Johnson YJ, Myinit MS, Herrmann JA, Mathewson A, Maddox CW, Troutt HF, Opacich K. Preliminary findings on the prevalence of methicillin-resistant Staphylococcus species in healthy horses and their human contacts. Silver Spring: United States Food and Drug Administration; 2007. 128.

7. Ruthland BE, Weese JS, Bolin C, Au J, Malani AN. Human-to-dog transmission of methiciliilin-resistant Staphylococcus aureus. Emerg Infect Dis. 2009:15:1328.

8. Van Duijkeren E, Ten Horn L, Wagenaar JA, De Bruijn M, Laarhoven L, Verstappen K, et al. Suspected horse-to-human transmission of MRSA ST398. Emerg Infect Dis J. 2011;17:1137.

9. Bramble M, Morris D, Tolomeo P, Lautenbach E. Potential role of pet animals in household transmission of Methicillin-resistant Staphylococcus aureus: a narrative review. Vector Borne Zoonotic Dis. 2011;11(6):617-20.

10. Bergstrom K, Bengtsson B, Nyman A, Andersson UG. Longitudinal study of horses for carriage of methicillin-resistant Staphylococcus aureus following wound infections. Vet Microbiol. 2013;163(3-4):388-91.

11. Carfora V, Caprioli A, Grossi I, Pepe M, Alba P, Lorenzetti S, Amoruso R, Sorbara L, Franco A, Battisti A. A methicillin-resistant Staphylococcus aureus (MRSA) sequence type 8, spa type t11469 causing infection and colonizing horses in Italy. Pathog Dis. 2016;74(4):ftw025.

12. Kuroda T, Kinoshita Y, Niwa H, Shinzaki Y, Tamura N, Hobo S, Kuwano A. Meticillin-resistant Staphylococcus aureus colonisation and infection in thoroughbred racehorses and veterinarians in Japan. Vet Rec. 2016;178(19): 473-U444.

13. Schwaber MJ, Navon-Venezia S, Masarwa S, Tirosh-Levy S, Adler A, Chmelnitsky I, Carmeli Y, Klement E, Steinman A. Clonal transmission of a rare methicillinresistant Staphylococcus aureus genotype between horses and staff at a veterinary teaching hospital. Vet Microbiol. 2013;162(2-4):907-11.

14. van Spijk JN, Schoster A, Wittenbrink MM, Schmitt S. A retrospective analysis of antimicrobial resistance in bacterial pathogens in an equine hospital (2012-2015). Schweiz Arch Tierheilkd. 2016;158(6):433-42.

15. National Committee for Clinical Laboratory Standards. Performance standards for antimicrobial disk susceptibility tests, approved standard M2A5, vol. 14. 5th ed; 1993

16. National Committee for Clinical Laboratory Standards: Performance standards for antimicrobial disk susceptibility tests - fifth informational supplement. Approved standard M100-S5, vol. 14; 1994

17. National Committee for Clinical Laboratory Standards. Performance standards for antimicrobial susceptibility tests. Approved standard M2-A6. 6th ed; 1997.

18. National Committee for Clinical Laboratory Standards. Methods for dilution antimicrobial susceptibility tests for bacteria that grow aerobically. 3rd ed; 1997.

19. National Committee for Clinical Laboratory Standards: Performance standards for antimicrobial susceptibility testing. Approved standard, M100-S8. 1997.

20. SAS Institute. Base SAS 9.4 Procedures Guide. Cary: SAS Institute; 2014

21. Magiorakos AP, Srinivasan A, Carey RB, Carmeli Y, Falagas ME, Giske CG, Harbarth S, Hindler JF, Kahlmeter G, Olsson-Liljequist B, et al. Multidrugresistant, extensively drug-resistant and pandrug-resistant bacteria: an international expert proposal for interim standard definitions for acquired resistance. Clin Microbiol Infect. 2012:18(3):268-81.

22. Theelen MJP, Wilson WD, Edman JM, Magdesian KG, Kass PH. Temporal trends in in vitro antimicrobial susceptibility patterns of bacteria isolated from foals with sepsis: 1979-2010. Equine Vet J. 2014;46(2):161-8.

23. Leekha S, Diekema DJ, Perencevich EN. Seasonality of staphylococcal infections. Clin Microbiol Infect. 2012;18(10):927-33.
24. van Balen J, Mowery J, Piraino-Sandoval M, Nava-Hoet RC, Kohn C, Hoet AE. Molecular epidemiology of environmental MRSA at an equine teaching hospital: introduction, circulation and maintenance. Vet Res. 2014;45:17.

25. Aslantas O, Turkyilmaz S, Yilmaz MA, Erdem Z, Demir C. Isolation and molecular characterization of methicillin-resistant staphylococci from horses, personnel and environmental sites at an equine hospital in Turkey. J Vet Med Sci. 2012;74(12):1583-8.

26. Van den Eede A, Martens A, Lipinska U, Struelens M, Deplano A, Denis O, Haesebrouck F, Gasthuys F, Hermans K. High occurrence of methicillinresistant Staphylococcus Aureus ST398 in equine nasal samples. Vet Microbiol. 2009;133(1):138-44.

27. Abbott $Y$, Leggett B, Rossney AS, Leonard FC, Markey BK. Isolation rates of meticillin-resistant Staphylococcus aureus in dogs, cats and horses in Ireland. Vet Rec. 2010;166(15):451-5.

28. Axon JE, Carrick JB, Barton MD, Collins NM, Russell CM, Kiehne J, Coombs G. Methicillin-resistant Staphylococcus aureus in a population of horses in Australia. Aust Vet J. 2011;89(6):221-5.

29. Burton S, Reid-Smith R, McClure JT, Weese JS. Staphylococcus aureus colonization in healthy horses in Atlantic Canada. Can Vet J. 2008;49(8):797-9.

30. Chambers HF, FR DL. Waves of resistance: Staphylococcus aureus in the antibiotic era. Nat Rev Microbiol. 2009;7(9):629-41.

31. Priyantha R, Gaunt MC, Rubin JE. Antimicrobial susceptibility of staphylococcus pseudintermedius colonizing healthy dogs in Saskatoon, Canada. Can Vet J. 2016;57(1):65-9.

32. Schnellmann C, Gerber $V$, Rossano A, Jaquier $V$, Panchaud Y, Doherr MG, Thomann A, Straub R, Perreten V. Presence of new mecA and mph (C) variants conferring antibiotic resistance in staphylococcus spp. isolated from the skin of horses before and after clinic admission. J Clin Microbiol. 2006; 44(12):4444-54.

33. Haenni M, Targant H, Forest K, Sevin C, Tapprest J, Laugier C, Madec JY. Retrospective study of necropsy-associated coagulase-positive staphylococci in horses. J Vet Diagn Investig. 2010;22(6):953-6.

34. Cuny C, Abdelbary MMH, Köck R, Layer F, Scheidemann W, Werner G, Witte W. Methicillin-resistant Staphylococcus aureus from infections in horses in Germany are frequent colonizers of veterinarians but rare among MRSA from infections in humans. One Health. 2016;2:11-7.

35. Klimienė I, Ružauskas M, Mockeliūnas R, Šiugždinienè R, Virgailis M, Špakauskas V. Antimicrobial resistance of Staphylococcus spp. isolated from riding-horses nasal mucosa. Veterinarija ir Zootechnika. 2015;71(93):32-38

36. Toombs-Ruane LJ, Riley CB, Kendall AT, Bolwell CF, Benschop J, Rosanowski SM. Antimicrobial susceptibilities of aerobic isolates from respiratory samples of young New Zealand horses. J Vet Intern Med. 2015;29(6):1700-6.

37. Tiwari R, Yadav SK, Singh S. Methicillin resistant Staphylococcus aureus isolated from wounds of livestock and companion animals of Uttar Pradesh India: a preliminary study. Int J Pharmacol. 2016;12(8):821-9.

38. Sieber S, Gerber V, Jandova V, Rossano A, Evison JM, Perreten V. Evolution of multidrug-resistant Staphylococcus aureus infections in horses and colonized personnel in an equine clinic between 2005 and 2010. Microb Drug Resist. 2011;17(3):471-8.

39. Tokateloff N, Manning ST, Weese JS, Campbell J, Rothenburger J, Stephen C, Bastura V, Gow SP, Reid-Smith R. Prevalence of methicillin-resistant Staphylococcus aureus colonization in horses in Saskatchewan, Alberta, and British Columbia. Can Vet J. 2009:50(11):1177-80

40. Weese JS, Archambault M, Willey BM, Dick H, Hearn R, Kreiswirth BN, SaidSalim B, McGeer A, Likhoshvay Y, Prescott JF, et al. Methicillin-resistant Staphylococcus aureus in horses and horse personnel, 2000-2002. Emerg Infect Dis. 2005;11(3):430-5.

41. Giguere S, Lee E, Williams E, Cohen ND, Chaffin MK, Halbert N, Martens RJ, Franklin RP, Clark CC, Slovis NM. Determination of the prevalence of antimicrobial resistance to macrolide antimicrobials or rifampin in Rhodococcus equi isolates and treatment outcome in foals infected with antimicrobialresistant isolates of R equi. J Am Vet Med Assoc. 2010;237(1):74-81.

42. Marsh PS, Palmer JE. Bacterial isolates from blood and their susceptibility patterns in critically ill foals: 543 cases (1991-1998). J Am Vet Med Assoc. 2001:218(10):1608-10.

43. Russell CM, Axon JE, Blishen A, Begg AP. Blood culture isolates and antimicrobial sensitivities from 427 critically ill neonatal foals. Aust Vet J. 2008;86(7):266-71.

44. Theelen MJ, Wilson WD, Edman JM, Magdesian KG, Kass PH. Temporal trends in prevalence of bacteria isolated from foals with sepsis: 1979-2010. Equine Vet J. 2014;46(2):169-73. 
45. Lowy FD. Antimicrobial resistance: the example of Staphylococcus aureus. J Clin Investig. 2003;111(9):1265-73.

46. Guignard B, Entenza JM, Moreillon P. Beta-lactams against methicillinresistant staphylococcus aureus. Curr Opin Pharmacol. 2005;5(5):479-89.

47. Sahebnasagh R, Saderi H, Owlia P. The prevalence of resistance to Methicillin in Staphylococcus aureus strains isolated from patients by PCR method for Detec-tion of mecA and nuc genes. Iran J Public Health. 2014 43(1):84-92.

48. Weese JS, Rousseau J. Attempted eradication of methicillin-resistant Staphylococcus aureus colonisation in horses on two farms. Equine Vet J. 2005:37(6):510-4.

Submit your next manuscript to BioMed Central and we will help you at every step:

- We accept pre-submission inquiries

- Our selector tool helps you to find the most relevant journal

- We provide round the clock customer support

- Convenient online submission

- Thorough peer review

- Inclusion in PubMed and all major indexing services

- Maximum visibility for your research

Submit your manuscript at www.biomedcentral.com/submit
Biomed Central 